\title{
Bedside ultrasound reliability in locating catheter and detecting complications
}

Payman Moharamzadeh ${ }^{1}$, Ali Taghizadieh ${ }^{1}$, Mahboob Pouraghaei ${ }^{1}$, Dariush Taghiloo ${ }^{2}$, Samad Shams Vahdati ${ }^{1}$, Kavous Shahsavari $\mathrm{Nia}^{3 *}$, Abbasali Dehghani ${ }^{4}$

1. Department of Emergency medicine, Tabriz University of Medical Sciences, Tabriz, Iran

2. Department of Emergency Medicine, Zanjan University of Medical sciences, Zanjan, Iran

3. Road Traffic Injury Research Center, Tabriz University of Medical Sciences, Tabriz, Iran

4. Department of Anesthesiology, Tabriz University of Medical Sciences, Tabriz, Iran

\begin{abstract}
Introduction: Central venous catheterization is one of the most common medical procedures and is associated with such complications as misplacement and pneumothorax. Chest X-ray is among good ways for evaluation of these complications. However, due to patient's excessive exposure to radiation, time consumption and low diagnostic value in detecting pneumothorax in the supine patient, the present study intends to examine bedside ultrasound diagnostic value in locating tip of the catheter and pneumothorax. Materials and methods: In the present cross-sectional study, all referred patients requiring central venous catheterization were examined. Central venous catheterization was performed by a trained emergency medicine specialist, and the location of catheter and the presence of pneumothorax were examined and compared using two modalities of ultrasound and x-ray (as the reference standard). Sensitivity, specificity, and positive and negative predicting values were reported. Results: A total of 200 non-trauma patients were included in the study ( $58 \%$ men). Cohen's Kappa consistency coefficients for catheterization and diagnosis of pneumothorax were found as $0.49(95 \% \mathrm{Cl}$ : 0.43-0.55), 0.89 ( $\mathrm{P}<0.001),(95 \% \mathrm{Cl}: 97.8-100)$, respectively. Also, ultrasound sensitivity and specificity in diagnosing pneumothorax were $75 \%$ (95\% Cl: $35.6-95.5)$, and 100\% (95\% Cl: $97.6-$ 100), respectively. Conclusion: The present study results showed low diagnostic value of ultrasound in determining catheter location and in detecting pneumothorax. With knowledge of previous studies, the search still on this field.
\end{abstract}

Keywords: Central venous catheterization; complications; bedside ultrasound; radiography;

\section{Corresponding author:}

Kavous Shahsavari Nia, MD

Road Traffic Injury Research Center, Tabriz University of Medical Sciences, Tabriz, Iran

Postal code: 5166614756 Phone/Fax: +989125597918. Email: kavous.shahsavari@yahoo.com

Receive date: 2016-09-20| Accept date: 2016-10-15| Publish date: 2016-11-12

DOI: 10.7575/aiac.abcmed.16.04.04.03 


\section{Introduction}

Central Venous Catheterization (CVC) is one of the most important elective medical procedures for critically ill patients that can be used in hemodynamic monitoring, administration and dosage control of medication (1). CVC is one of the most common medical procedures, in that according to statistics, more than 5 million catheters are placed annually in the United States $(2,3)$. However, catheterization is associated with complications like misplacement and pneumothorax, which has been reported from $3.3 \%$ to $14 \%(4,5)$. Although, there is still controversy about location of central venous catheter tip, new guidelines identify the upper vena cava junction at the right atrium as the optimal location, and suggest radiography as the reference standard for its assessment $(5,6)$.

Patient's excessive exposure to radiation is the most important limitation in chest radiography. Moreover, some studies argue that accuracy and credibility of radiography in locating the catheter has been overestimated $(7,8)$. Thus, researchers are seeking other evaluation techniques that are highly accurate, reliable and low cost, and expose the patient to less radiation.

A low-cost and harmless method is bedside ultrasound. Recent studies have shown that bedside ultrasound can be useful in management of critically ill patients $[9,10]$. In this respect, Vezzani et al. recommend ultrasound as another technique for evaluating placement and pneumothorax, and argue that ultrasound has a more diagnostic value in pneumothorax diagnosis than radiography, and is less costly for patients (11). Furthermore, Maury et al. (12) and Lichtenstein et al. (13) also found similar results, and argue that ultrasound may provide a more accurate test for pneumothorax monitoring compared to chest radiography. Ultrasound is also not without limitations. For example, absence of ultrasonic windows, and presence of a wound in the chest or obesity in some patients renders ultrasound is more challenging to perform [14]. Also, operator's expertise significantly affects interpretation of ultrasound results. A study conducted by Cortellaro et al. in 2014 showed sensitivity of ultrasound of $33 \%$ in locating central venous catheter (15). These limitations have led to controversy about bedside ultrasound as a diagnostic modality in locating catheter, and its complications. Accordingly, the present study intends to examine diagnostic value of bedside ultrasound in determining location of catheter tip and its subsequent pneumothorax.

\section{Materials and methods}

Study design and setting:

This cross-sectional study enrolled patients referred to Imam Reza Hospital in Tabriz-Iran, over a 12-month period from April 2011 to March 2012. The present study protocol was approved by the ethics committee of Tabriz University of Medical Sciences. During the study period, researchers complied with the principles of Helsinki Convention. Patients or their company completed informed consent form for participation before entering the study.

\section{Participating patients:}

Study population consisted of patients referred to Imam Reza teaching hospital requiring central venous catheterization. Inclusion criteria were older than 18 years old, and candidate for central venous catheterization or dialysis catheter. Exclusion criteria included patients with known anatomic cardiac impairments, patients with cardiac shunts, and pregnant patients.

Catheterization indication was determined by physicians not involved in the study, based on medical requirement. 


\section{Procedure:}

Central Venous Lines (CVL Catheters) were placed by an emergency medicine specialist, trained in ultrasound catheterization and pneumothorax diagnosis. In the present study, the following catheters were used: two lumen hemodialysis catheterization set (Arrow, USA$20 \mathrm{~cm}, 12$ French), and two lumen central venous catheterization set (Arrow, USA- $20 \mathrm{~cm}$, 8 French), which were placed percutaneously using Seldinger technique. Placement was performed according to anatomic landmark, without fluoroscopy or guided ultrasound in supine position. Following placement, location of catheter and also presence of pneumothorax were studied using two modalities of ultrasound (LOGIO 200, PRO Series Ultra Sonography, $10-15 \mathrm{~Hz}$ ) and chest radiography.

Misplacement of catheter was defined as positioning of catheter tip in the right atrium or venous other than the superior vena cava. To that end, for ultrasound evaluation prior to fixing catheter to the wall, catheter placement assessment was carried out using injection of ready-made agitated (aspirated) normal saline, which is a standard techniques recommended by the European Society of Neurosonology and Cerebral Hemodynamics [16]. First, the cardiac subxiphoid image was observed using bedside ultrasound as saline solution $(9 \mathrm{ml}$ of saline and $1 \mathrm{ml}$ of air) was injected through central catheter simultaneously. Assurance of placement of catheter in central venous was achieved through observation of air dissolved in the saline (air bubbling) in the right atrium and immediately in the right ventricle in cardiac image that manifests itself in the form of temporary increase in echogenicity in cardiac cavity. When layers of micro-bubbles are observed in the right atrium within 1 to 2 second of injection, placement is assumed correct, otherwise it is considered incorrect. Then, using surface ultrasound probe (11-16), the 2 nd to 5 th intercostal space from mid- clavicular lines to mid-auxiliary line was examined for potential pneumothorax $(17,18)$. Then, immediately catheter was fixed in position, and after dressing, simple chest radiography was performed as the golden standard (19). Then, second ultrasound was performed to evaluate misplacement and complication of catheterization. Interpretation of radiography was performed by a radiologist, blind to ultrasound results and purpose

Statistical analysis:

Data were analyzed using STATA-11 statistics program. Given sensitivity of $95 \%$, confidence of $95 \%(\alpha=0.05)$, and power of $90 \%(\beta=0.1)$, minimum sample size was found 183 patients (this article). Ultrasound and chest radiographic results were compared and presented as true positive and true negative, false positive and false negative. Then sensitivity, specificity, and positive and negative predicting value of ultrasound in detecting misplacement of catheter and pneumothorax, based on chest radiography results, were presented. It should be noted that level of agreement between two modalities was assessed by calculating Cohen's Kappa coefficient in 95\% confidence range.

\section{Results}

A total of 200 non-trauma patients, aged between 23 and 87 years, with mean age of $63.7 \pm 22.5$ years participated in this study $(58 \%$ men). Dialysis catheter was used for 71 (35.5\%) and central venous catheter for 129 (64.5\%). Radiographic findings showed 197 (98.7\%) of catheters were placed correctly (70 dialysis and $127 \mathrm{CVL}$ ). In other words, success rate was $98.5 \%$. Also, pneumothorax occurred in 8 cases (4\%), 3 of whom (4.2\%) had dialysis catheter and 5 (3.9\%) had CVL (P=0.94). Ultrasound diagnosed 1 (33.3\%) of the 3 misplaced cases, which means that ultrasound evaluation had 2 (66.7\%) false negative cases. This test was also able to diagnose $6(75 \%)$ of the 8 pneumothorax cases. 
The area under ROC curve for locating catheter position was found $0.67(95 \% \mathrm{Cl}$ : 0.340.99), which is indicative of low adequacy of ultrasound in this area (figure 1). Cohen's Kappa coefficient for ultrasound to diagnose catheter position was 0.49 (95\% Cl: 0.43-0.55, $\mathrm{p}<0.001)$. Also, sensitivity and specificity of ultrasound in locating catheter position were $33.3 \%(95 \% \mathrm{Cl}: 1.76-87.5)$ and $100 \%(95 \% \mathrm{Cl}$ : 97.8-100), respectively (Table 1 ).
The area under ROC curve of ultrasound to diagnose pneumothorax was $0.87(95 \% \mathrm{Cl}$ : 0.71-1.0) (Figure 1). Kappa coefficient obtained in detecting pneumothorax was 0.85 (95\% Cl: $0.677-1.027 ; \mathrm{P}<0.001)$. Also, sensitivity and specificity of ultrasound in detecting pneumothorax were $75 \%$ (95\% Cl: 35.6-95.5) and $100 \%(95 \% \mathrm{Cl}: 97.6-100)$, respectively (Table 1).

\begin{tabular}{|c|c|c|c|c|}
\hline \multirow[t]{2}{*}{ Diagnostic parameter } & \multicolumn{2}{|c|}{ Catheter position } & \multicolumn{2}{|c|}{ Pneumothorax } \\
\hline & Value (\%) & $\mathrm{Cl} 95 \%$ & Value (\%) & $\mathrm{Cl} 95 \%$ \\
\hline Sensitivity & 33.3 & $1.76-87.5$ & 75 & $35.6-95.5$ \\
\hline Specificity & 100 & $97.8-100$ & 100 & 97.6-100 \\
\hline Positive predicting value & 100 & $5.5-100$ & 100 & $51.7-100$ \\
\hline Negative predicting value & 99 & $96.0-99.8$ & 99 & $95.9-99.8$ \\
\hline Positive Likelihood ratio & --- & --- & --- & --- \\
\hline Negative Likelihood ratio & 0.67 & 0.3-1.48 & 0.25 & $0.08-0.83$ \\
\hline
\end{tabular}

Table 1: Ultrasound diagnostic value in locating catheter position and detecting pneumothorax

\section{Discussion}

The present study results showed that ultrasound had $100 \%$ specificity in detecting catheter position, but had a low sensitivity (33.3\%). This test had $75 \%$ sensitivity and $100 \%$ specificity in identifying pneumothorax. Accordingly, it seems, despite its high specificity in locating catheter placement and subsequent pneumothorax, ultrasound is not an appropriate alternative to radiography.

For technical reasons, radiography is not very reliable in pneumothorax evaluation, since chest radiography immediately after catheter placement leads to insufficient time for spread of pneumothorax, and pneumothorax does not sufficiently progress to be detectable $(20,21)$. Furthermore, chest radiography in anteriorposterior view has a low sensitivity in identifying latent pneumothorax, since air initially accumulates in the chest medial area, where radiography is unable to accurately evaluate this region in supine position (22). Recent guidelines strongly emphasize that catheter tip should not be placed in the heart, or be able to migrate to the heart $(7,19)$. These 


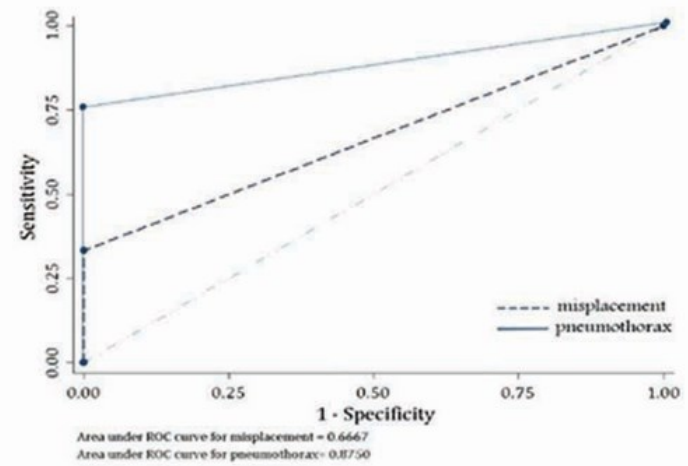

Figure 1: ROC ultrasound curve for detecting catheter position and pneumothorax

guidelines present space between superior vena cava and right atrium as the best catheter position. Thus, it is recommended that portable radiography be used for critically ill patients, despite the high costs and patient and physician exposure to radiation (23). However, it should be borne in mind that superior vena cava junction at right atrium is not visible with portable radiography, and cause false positive results (misplacement) to be reported in $47 \%$ of cases (24). All these limitations have led to recent studies to seek a reliable method to reduce complications caused by catheter placement. Existing evidence suggests effective role of ultrasound in correct catheter placement (ultrasound guided). During 19962003 three meta-analyses provided strong evidence for use of ultrasound guided central venous catheterization (25-27). But, none of these meta-analyses had been performed on critically ill or emergency patients. All three of these meta-analyses show that use of this method can cause improved success rate, reduce number of catheterization attempts and complications. Also, this technique leads to reduced medical costs. However, in these studies, efficacy of use of ultrasound guided has not been assessed for mortality rate, hospitalization period, or long term complications. Moreover, ultrasound guided also has limitations that have led to its low use by physicians despite all its advantages (28). This technique can only be effective in catheterization and cannot detect pneumothorax. Effective use of ultrasound to the diagnosis of pneumothorax and misplacement of catheter requires a good knowledge of the anatomy and pathophysiology of the pulmonary system. In addition, all ultrasound examinations are known to be operator dependent. For this reason several studies demonstrated that the overall sensitivity of ultrasonography for the diagnosis of pneumothorax varied from $58.9 \%$ to $100 \%(29,30)$. These reasons justify difference among the studies.

To compare findings of present study, a study by Vezani et al. can be cited, which argues that sensitivity and specificity of ultrasound to confirm catheter position are $96 \%$ and $93 \%$ respectively. Also, sensitivity of this diagnostic test in pneumothorax evaluation was reported 98\% (11). Zanobetti et al. showed high adaptability of ultrasound and radiography in assessment of catheterization (94\% sensitivity and $89 \%$ specificity) and occurrence of pneumothorax (100\% sensitivity) (31). However, Cortellaro et al. showed Contrast Enhanced Sonography sensitivity of 33\% and specificity of $98 \%$ in detecting catheter position (15). It can be seen that there is a huge difference of opinion about ultrasound sensitivity and specificity in determining catheter position, which requires further research.

Emergency physicians can do all of emergent procedures in emergency department and have this ability and have high degree performance (32); also, presence of Ultrasonography instruments can help to do procedures beside patient evaluation specially in trauma patients $(33,34)$ but complication can happen in emergency situation such as malpositioning 
(35); we must know when malpositioning occurred, specially when catheter presents in arteria, emergency physicians must not withdraw it before well evaluation and surgeon presentation (36).

In the present study, all ultrasounds were performed by one person, which prevents interobservation. However, among study limitations, the role of ultrasound operator's dexterity may have affected the results. Perhaps, the reason for low sensitivity in determining catheter position was due to this fact. It should also be mentioned that in venous catheterization, observing air bubbles in the heart is not necessarily indicative of catheter placement in superior vena cava or right atrium, since when catheter is misplaced (for instance in the subclavian vein), by injection of saline containing gas bubbles, air bubbles are observed at the right atrium junction with SVC due to blood circulation. Yet, very low frequency of unsuccessful catheter placement (1.5\%) may have affected sensitivity of ultrasound and created false low sensitivity.

\section{Conclusion}

Study results indicate low ultrasound sensitivity in catheterization. This test has 75\% sensitivity and $100 \%$ specificity in detecting pneumothorax. The present study show that ultrasound cannot be a suitable alternative to radiography in determining catheter position and detecting pneumothorax. Thus, with knowledge of previous studies, the search still on this field.

\section{References}

1. Graham AS, Ozment C, Tegtmeyer K, Lai S, Braner DAV. Central Venous Catheterization. New England Journal of Medicine. 2007;356(21):e21.

2. Taylor RW, Palagiri AV. Central venous catheterization. Critical care medicine. 2007;35(5):1390-6.

3. Agee K, Balk R. Central venous catheterization in the critically ill patient. Critical care clinics. 1992;8(4):677.

4. Pikwer A, Baath L, Davidson B, Akeson J. The incidence and risk of central venous catheter malpositioning: a prospective cohort study in 1619 patients. Anaesthesia and intensive care. 2008;36(1):30-7.

5. Gladwin MT, Slonim A, Landucci DL, Gutierrez DC, Cunnion RE. Cannulation of the internal jugular vein: is postprocedural chest radiography always necessary? Critical care medicine. 1999;27(9):1819-23.

6. Wirsing M, Schummer C, Neumann R, Steenbeck J, Schmidt P, Schummer W. Is traditional reading of the bedside chest radiograph appropriate to detect intraatrial central venous catheter position? CHEST Journal. 2008;134(3):52733.

7. Fletcher S, Bodenham A. Safe placement of central venous catheters: where should the tip of the catheter lie? British journal of anaesthesia. 2000;85(2):188-91.

8. Matsushima K, Frankel HL. Bedside ultrasound can safely eliminate the need for chest radiographs after central venous catheter placement: CVC sono in the surgical ICU (SICU). Journal of Surgical Research. 2010;163(1):155-61.

9. Beaulieu Y, Marik PE. Bedside Ultrasonography in the ICUPart 1. CHEST Journal. 2005;128(2):881-95. 
10. Beaulieu Y. Bedside echocardiography in the assessment of the critically ill. Critical care medicine. 2007;35(5):S235-S49.

11. Vezzani A, Brusasco C, Palermo S, Launo C, Mergoni M, Corradi F. Ultrasound localization of central vein catheter and detection of postprocedural pneumothorax: An alternative to chest radiography*. Critical care medicine. 2010;38(2):533-8.

12. Maury E, Guglielminotti J, Alzieu M, Guidet B, Offenstadt G. Ultrasonic examination: an alternative to chest radiography after central venous catheter insertion? American journal of respiratory and critical care medicine. 2001;164(3):403-5.

13. Lichtenstein DA, Mezière G, Lascols N, Biderman P, Courret J-P, Gepner A, et al. Ultrasound diagnosis of occult pneumothorax*. Critical care medicine. 2005;33(6):1231-8.

14. Ammirati C, Maizel J, Slama M. Is chest X-ray still necessary after central venous catheter insertion?*. Critical care medicine. 2010;38(2):715-6.

15. Cortellaro F, Mellace L, Paglia S, Costantino G, Sher S, Coen D. Contrast enhanced ultrasound vs chest X-ray to determine correct central venous catheter position. The American journal of emergency medicine. 2014;32(1):78-81.

16. Ghadiali N, Teo LM, Sheah K. Bedside confirmation of a persistent left superior vena cava based on aberrantly positioned central venous catheter on chest radiograph. British Journal of Anaesthesia. 2006;96(1):53-6.

17. Lichtenstein D, Meziere G, Biderman P, Gepner A. The comet-tail artifact: an ultrasound sign ruling out pneumothorax. Intensive care medicine. 1999;25(4):383-8.

18. Lichtenstein DA, Menu Y. A Bedside Ultrasound Sign Ruling Out Pneumothorax in the Critically III Lung Sliding. CHEST Journal. 1995;108(5):1345-8.

19. Food and Drug Administration. Precautions Necessary With Central Venous Catheter. Washington, DC, US Government Printing Office. 1989: pp 15-16.

20. Soldati G, Testa A, Sher S, Pignataro G, La Sala M, Silveri NG. Occult Traumatic PneumothoraxDiagnostic Accuracy of Lung Ultrasonography in the Emergency Department. CHEST Journal. 2008;133(1):204-11.

21. Zhang M, Liu Z-H, Yang J-X, Gan J-X, Xu S-W, You X-D, et al. Rapid detection of pneumothorax by ultrasonography in patients with multiple trauma. Critical Care. 2006;10(4):R112.

22. Ball CG, Kirkpatrick AW, Laupland KB, Fox DL, Litvinchuk S, Dyer DM, et al. Factors related to the failure of radiographic recognition of occult posttraumatic pneumothoraces. The American journal of surgery. 2005;189(5):5416.

23. Webb W. Pulmonary edema, the acute pulmonary distress syndrome and radiology in the intensive care unit. In: Thoracic Imaging: Pulmonary and Cardiovascular Radiology. Webb W (Ed). Lippincott Williams \& Wilkins, Philadelphia 2005:331-55.

24. Reynolds N, McCulloch AS, Pennington CR, MacFadyen RJ. Assessment of distal tip position of long-term central venous feeding catheters using transesophageal echocardiology. Journal of Parenteral and Enteral Nutrition. 2001;25(1):39-41.

25. Hind D, Calvert N, McWilliams R, Davidson A, Paisley S, Beverley C, et al. Ultrasonic locating devices for central venous cannulation: meta-analysis. Bmj. 2003;327(7411):361.

26. Keenan SP. Use of ultrasound to place central lines. Journal of critical care. 2002;17(2):126-37.

27. Randolph AG, Cook DJ, Gonzales CA, Pribble CG. Ultrasound guidance for placement of central venous catheters: a meta-analysis of the literature. Critical care medicine. 1996;24(12):2053-8.

28. Matera JT, Egerton-Warburton D, Meek R. Ultrasound guidance for central venous catheter placement in Australasian emergency departments: potential barriers to more widespread use. Emergency Medicine Australasia. 2010;22(6):514-23. 
29. Alsalim, W. and D. Lewis. Towards evidence based emergency medicine: Best BETs from the Manchester Royal Infirmary. BET 1: Is ultrasound or chest $x$ ray best for the diagnosis of pneumothorax in the emergency department? Emerg Med J. 2009; 26(6): 434-435.

30. Heydari F, Esmailian M, Dehghanniri M. Diagnostic Accuracy of Ultrasonography in the Initial Evaluation of Patients with Penetrating Chest Trauma. Emergency. 2014;2(2): 81-84.

31. Zanobetti M, Coppa A, Bulletti F, Piazza S, Nazerian P, Conti A, et al. Verification of correct central venous catheter placement in the emergency department: comparison between ultrasonography and chest radiography. Internal and emergency medicine. 2012:1-8.

32. AMIRI H, VAHDATI SS, GHODRAT N, SOHRABI A, MARZABADI LR, Garadagh A. Emergency Medicine Physicians can Manage all Emergent Procedures in the Emergency Department. Turkish Journal of Emergency Medicine. 2009;9(3):101-4.

33. Ala AR, Pouraghaei M, Vahdati SS, Taghizadieh A, Moharamzadeh P, Arjmandi H. Diagnostic Accuracy of Focused Assessment With Sonography for Trauma in the Emergency Department. Trauma Monthly. 2016 Jun;21(4).

34. Tajoddini S, Vahdati SS. Ultrasonographic diagnosis of abdominal free fluid: accuracy comparison of emergency physicians and radiologists. European Journal of Trauma and Emergency Surgery. 2013 Feb 1;39(1):9-13.

35. Fadaei Haghi A, Shokri R, Shams Vahdati S, Darei M, Eftekhar Milani F, Tajoddini S. A catheter malpositioned patient with pain and paresthesia. Journal of Emergency Practice and Trauma. 2015;1(3).

36. Vahdati SS. Should a Double-Lumen Catheter be withdrawn?. Journal of cardiovascular and thoracic research. 2011;3(3):97. 